\title{
INVESTIGATING THEORETICALLY AND EXPERIMENTALLY TH EFFECT OF A MOVABLE LATERAL SILL ON HYDRAULIC JUMP
}

Amany A. Habib, Mohamed A. Nassar

\begin{abstract}
There is no doubt that, reducing of the hydraulic jump length and improving its ability to disperse energy $\mathrm{i}$ main outputs of the modification processes of the jump. The idea is to set up a Movable Lateral Sill (MLS). be a hollow gate downstream the main gate. The properties of MSL have been studied experimentally and this paper investigated the effects of different relative positions (x/G), relative heights (Hs/G), relative hei of the sill $(\mathrm{Hob} / \mathrm{G})$ and contraction coefficient $C(C=[b-b c] / b)$. It was found that, the use of MSL improvı characteristics. Moreover, it was found that the sill of $x / G=2.31, \mathrm{Hs} / \mathrm{G}=0.7, \mathrm{Hob} / \mathrm{G}=0.263$ and $\mathrm{C}=0.12$ s optimum results for the investigated ranges. It reduces the relative depth and length of the jump and incri relative energy loss by about 35\% in comparison with the classical jump. Two theoretical models are devel predict the relative depth and energy loss of the jump. Moreover, statistical equations for the different jum characteristics were developed using the multiple linear regression. A good agreement was obtained for th and statistical models. Finally, the results of this study may be recommended in engineering applications
\end{abstract}

